\title{
Post-13-Valent Pneumococcal Conjugate Vaccine Dynamics in Young Children of Serotypes Included in Candidate Extended- Spectrum Conjugate Vaccines
}

Shalom Ben-Shimol, Noga Givon-Lavi, Leore Kotler, Bart Adriaan van der Beek, David Greenberg, Ron Dagan

After worldwide implementation of 10-valent and 13-valent pneumococcal conjugate vaccines (PCV10/PCV13), a 20 -valent PCV (PCV20) was developed. We assessed dynamics of non-PCV13 additional PCV20 serotypes (VT20-13), compared with all other non-VT20 serotypes, in children <2 years of age in late PCV13 (2015-2017) and early PCV (2009-2011) periods. Our prospective population-based multifaceted surveillance included isolates from carriage in healthy children, children requiring chest radiography for lower respiratory tract infections (LRTIs), and children with non-LRTI illness, as well as isolates from acute conjunctivitis, otitis media (OM), and invasive pneumococcal disease (IPD). After PCV13 implementation, VT20-13 increased disproportionally in OM, IPD, and carriage in LRTI. VT20-13/non-VT20 prevalence ratio range was $0.26-1.40$. VT20-13 serotypes were more frequently antimicrobial-nonsusceptible than non-VT20 serotypes. The disproportionate increase of VT20-13 in respiratory infections and IPD points to their higher disease potential compared with all other non-VT20 as a group.

Streptococcus pneumoniae is a major cause of illness $\mathcal{S}$ and death worldwide $(1,2)$. It causes otitis media, sinusitis, pneumonia, and invasive pneumococcal diseases (IPD) (1).

Capsular polysaccharides are considered the most important virulence factor in S. pneumoniae (3). Currently, >95 capsular serotypes have been identified. Each serotype is distinguished by the chemical structure of

Author affiliations: Ben-Gurion University of the Negev, Beer Sheva, Israel (S. Ben-Shimol, N. Givon-Lavi, L. Kotler,

B.A. van der Beek, D. Greenberg, R. Dagan); Soroka University Medical Center, Beer Sheva (S. Ben-Shimol, N. Givon-Lavi,

L. Kotler, D. Greenberg)

DOI: https://doi.org/10.3201/eid2701.201178 its polysaccharides, serologic response, and other genetic characteristics (4). Pneumococcal capsule types are associated with pathogenic processes including complement deposition, inflammation, and binding to the C-type lectin of host phagocytes (3). Pneumococcal serotype appears to be important in determining colonization, disease development, and clinical phenotype. Indeed, in the time before pneumococcal conjugate vaccines (PCVs), a limited number of serotypes among the known $>95$ serotypes were responsible for $>70 \%$ of all IPD in children worldwide (5).

The routine use of PCVs in children worldwide has led to a decline of vaccine serotype (VT) IPD, mucosal diseases, and nasopharyngeal carriage (6-9). However, after PCV implementation, in spite of a decrease in overall pneumococcal disease rates, carriage of and disease from non-PCV serotypes (NVT) increased (5). As of October 2020, licensed PCVs contain 7 to 13 serotypes (7-valent [PCV7], 10-valent [PCV10], and 13-valent [PCV13]). Efforts to develop extended-spectrum (higher valency) PCVs have led to the development of 15- and 20-valent PCVs (PCV15, PCV20), both currently in advanced stages of clinical studies. The experimental PCV20 includes, beyond the 13 serotypes of PCV13 $(1,3,4,5,6 \mathrm{~A}, 6 \mathrm{~B}, 7 \mathrm{~F}, 9 \mathrm{~V}, 14,19 \mathrm{~A}, 19 \mathrm{~F}, 18 \mathrm{C}$, and $23 \mathrm{~F})$, the additional pneumococcal serotypes 8 , 10A, 11A, 12F, 15B/C, 22F and 33F (10), of which 2 (serotypes $22 \mathrm{~F}$ and $33 \mathrm{~F}$ ) are also contained in PCV15 (11). These additional PCV20 serotypes (VT20-13) have been increasingly observed in recent years as common IPD serotypes. However, data are scarce in regard to their relative role in other entities such as carriage and respiratory disease in young children.

We compared the proportion rate dynamics of the added PCV20 serotypes (VT20-13) with PCV13 (VT13) and remaining non-VT20 (NVT20) serotypes 
in nasopharyngeal carriage of healthy young children and in children with non-lower respiratory tract infections (LRTIs) (pediatric emergency room [PER] visits in which chest radiography was not done), lower respiratory diseases requiring chest radiography, IPD, and pneumococcal culture-positive acute conjunctivitis and otitis media $(\mathrm{OM})$.

\section{Materials and Methods}

\section{Study Design}

Our data derive from several ongoing, populationbased, active surveillance projects conducted by the Pediatric Infectious Disease Unit, Soroka University Medical Center (SUMC) during 2009-2017. We defined 2 periods: early PCV (2009-2011) and late PCV13 (2015-2017). SUMC is the only hospital in southern Israel providing healthcare to the entire region, which enables us to conduct population-based studies.

\section{Setting and Study Population}

During the study period, the average total number of annual births in the Negev district in southern Israel was $\approx 15,000$. Over $95 \%$ of the children in the district are born and receive medical services at the SUMC. In the Negev district, the Jewish and the Bedouin populations live side-by-side. The socioeconomic conditions and the lifestyles of the 2 groups differ, but both have access to the same medical services. The Jewish population, mainly urban, resembles developed populations, whereas the Bedouin population, formerly desert nomads in transition to a Western lifestyle, resembles developing populations, with a high occurrence of infectious diseases including pneumococcal disease and complex OM $(6,7,9)$. Contact between the children of the 2 populations is rare. During the study period, $\approx 50 \%$ of children $<2$ years of age in southern Israel were Jews and $50 \%$ were Bedouins. Our data derived from 5 prospective active surveillance projects.

\section{1) Carriage in Healthy Children (Group 1; Community Setting, Southern Israel)}

This study, initiated in 2011, included nasopharyngeal cultures obtained from healthy children $<2$ years of age who were brought to the maternal and child healthcare centers in southern Israel for vaccination. A nasopharyngeal swab specimen was obtained after parents gave written informed consent.

\section{2) Carriage during IIIness (Groups 2 and 3; Hospital PER Setting, Southern Israel)}

This study, initiated in 2009, included cultures obtained from the PER of SUMC. Each workday, healthcare workers obtained nasopharyngeal cultures from the first 4 Jewish and 4 Bedouin children who were $<2$ years of age, residents of the Negev, and brought to the PER for any reason (9). We defined 2 groups in that surveillance: carriage in nonLRTI (children seen at the PER for any disease except those necessitating chest radiography); and carriage in LRTI (children with LRTI from whom a chest radiography was obtained) (6).

\section{3) Conjunctivitis (Group 4; Both Community and Hospital Settings, Southern Israel)}

The study population included children $<24$ months of age who were residents of the Negev region, received a diagnosis of acute conjunctivitis in a community clinic or at SUMC (either PER or hospitalized), and had a conjunctival culture sent to the Clinical and Microbiology Laboratory of the SUMC since 2009.

\section{4) Otitis Media (Group 5; Both Community and Hospital} Settings, Southern Israel)

The study population included children $<24$ months of age who were residents of the Negev region and had OM judged to necessitate middle ear fluid (MEF) culture. Cultures were obtained by tympanocentesis or by swab of the external canal of children with acute (<7 days) spontaneous otorrhea. Most of the children had complex OM (nonresponsive, recurrent, spontaneous perforation, or chronic ear effusion) $(7,12,13)$. Children found to have pneumococcal MEF isolates during 2009-2017 were included (7).

\section{5) IPD (Group 6; Both Community and Hospital Settings, Israel, Nationwide)}

This nationwide study was conducted in all 27 medical centers routinely obtaining cerebrospinal fluid (CSF) and blood cultures from children <24 months old in Israel; sites included 26 hospitals and 1 outpatient health maintenance organization (14). This setting enabled us to cover all culture-confirmed IPD cases among the population of Israel and calculate national incidence. No CSF cultures and $<1 \%$ of blood cultures were obtained outside these centers. Data in our study were for IPD episodes identified since 2009.

\section{PCV Introduction to the Israeli National Immunization Plan and Uptake}

Israel implemented PCV7 and PCV13 vaccination during July 2009-November 2010 on a schedule of 2, 4, and 12 months; catch-up vaccines were also administered for PCV7 in children <2 years. Vaccine uptake evaluation methods were as previously described (14). By June $2011, \approx 80 \%$ of children $7-11$ months of age had 
received $\geq 2$ doses of PCV7, PCV13, or both, and $\approx 90 \%$ by December 2012; thereafter, $\approx 95 \%$ had received $\geq 2$ PCV13 doses. By June 2011, a total of 36\% of children 24-35 months of age had received $\geq 3$ PCV7/PCV13 doses; that number increased to $87 \%$ by December 2012 , and thereafter, $>90 \%$ received $\geq 3$ PCV13 doses.

\section{Bacteriology}

\section{Nasopharyngeal Cultures}

Nasopharyngeal samples were obtained as previously described (9). In brief, we used a flexible Dacrontipped swab, introduced through the nostrils. These swabs were inoculated into modified Stewart transport medium (Medical Wire and Equipment Co., Ltd, https:/ / www.mwe.co.uk) and were processed within 16 hours at SUMC's clinical microbiology laboratory. Material from swabs was plated on Columbia agar with $5 \%$ sheep blood and $5.0 \mathrm{mg} / \mathrm{mL}$ gentamicin and incubated for $48 \mathrm{~h}$.

We presumptively identified S. pneumoniae on the basis of the presence of a-hemolysis and inhibition by optochin; we confirmed the identity of the bacteria present by a positive slide agglutination test result (Phadebact; MKL Diagnostics AB, http:/ /www. mkldiagnostics.com).

\section{Conjunctivitis Cultures}

We enrolled in the study all patients $<24$ months of age who received a diagnosis of conjunctivitis from pediatricians at SUMC or at the primary clinical service in southern Israel and whose conjunctival swabs were cultured at SUMC's clinical microbiology laboratory and grew S. pneumoniae. Swabbing methods were described previously (15). Specimen swabs were placed in transport medium and were processed in a similar manner to the nasopharyngeal swabs.

\section{Otitis Media and Middle Ear Fluid Cultures}

Specimen swabs were sent in transport medium. They were processed in a similar manner to the nasopharyngeal and conjunctival swabs.

\section{IPD}

Pneumococcal isolates from blood and CSF were initially identified by each center using local standard procedures as described previously $(14,16)$.

\section{Serotypes}

All strains were serotyped by Quellung reaction using the antisera of Statens Serum Institute, Copenhagen, Denmark. Methods of specimen transport were described previously $(7,9,14)$.

\section{Antimicrobial Susceptibility Testing}

We performed antimicrobial susceptibility testing by Etest (AB Biodisk, http://www.abbiodisk. com) and Kirby-Bauer disk diffusion in accordance with Clinical Laboratory Standards Institute recommendations (17). Antimicrobial nonsusceptibility among isolates was defined as MIC above or a zone diameter below the susceptibility breakpoint. For penicillin, we defined 2 nonsusceptibility cutoff values: $\mathrm{MIC} \geq 0.1 \mathrm{mg} / \mathrm{L}$ and $\mathrm{MIC} \geq 1.0 \mathrm{mg} / \mathrm{L}$. We used 2 nonsusceptibility values because the low cutoff (MIC $\geq 0.1 \mathrm{mg} / \mathrm{L}$ ) indicates nonsusceptibility to oral penicillin (used for mucosal infections) and parenteral penicillin for CNS infections, whereas the high MIC (MIC $\geq 1.0 \mathrm{mg} / \mathrm{L}$ ) indicates the possibility of nonsusceptibility to parenteral penicillin for non-CNS infections. We used MIC $\geq 1.0 \mu \mathrm{g} / \mathrm{mL}$ for ceftriaxone. For all other antimicrobial drugs, we tested susceptibility by the Kirby-Bauer method: for erythromycin, a zone diameter of $\leq 21 \mathrm{~mm}$; for trimethoprim/sulfamethoxazole, $\leq 19 \mathrm{~mm}$; for tetracycline, $\leq 23 \mathrm{~mm}$; for chloramphenicol, $\leq 20 \mathrm{~mm}$; and for clindamycin, $\leq 19 \mathrm{~mm}$. Isolates nonsusceptible to $\geq 3$ of the antimicrobial categories were defined as multidrug resistant (MDR). In this study, we present antimicrobial susceptibility testing data for the late PCV13 period (2015-2017), for comparison of the current resistance patterns of VT20-13 versus the NVT20 serotype.

\section{Statistical Analysis}

We conducted statistical analysis using SPSS Statistics 25.0 software for Windows (https://www.ibm. com/analytics/spss-statistics-software); $p<0.05$ was considered statistically significant. We analyzed data from active surveillance during 2009-2017 by epidemiologic years, July through June. We calculated prevalence rate ratios (RRs) with 95\% CIs, comparing VT13, VT20-13, and NVT20 proportions in late PCV13 (2015-2017) versus early PCV (2009-2011) periods. Prevalence RRs were adjusted for age and ethnicity (Table 1, https://wwwnc.cdc.gov/EID/ article/27/1/20-1178-T1.htm; Figure 2).

\section{Results}

During the study period, a total of 9,089 isolates were analyzed: 2,638 from carriage in healthy children, 2,450 from carriage in non-LRTI, 1,819 from carriage in LRTI, 477 conjunctivitis, 756 OM, and 949 IPD isolates. Overall, throughout the study, $23.9 \%$ of all isolates were VT13, 23.3\% were VT20-13, and 52.7\% were NVT20 (Appendix Table 1, https://wwwnc. cdc.gov/EID/article/27/1/20-1178-App1.pdf). 
VT13, VT20-13 and NVT20 Proportions during the Early PCV Period

To enable better appreciation of disease potential of the 3 serotype groups (VT13, VT20-13 and NVT20) before PCV13 implementation, we analyzed the early PCV period separately. In the early PCV period, VT13 predominated in all groups. The proportions of VT13 of all isolates were $43.9 \%$ in carriage in non-LRTI, $52.1 \%$ carriage in LRTI, $45.1 \%$ conjunctivitis, $65 \%$ OM, and $71.8 \%$ IPD. Data for carriage in healthy children in this period were not available (Table 1; Figure 1).

For VT20-13, proportions were $17.8 \%$ for carriage in non-LRI, $13.4 \%$ in carriage in LRI, $20.5 \%$ conjuncti- vitis, $14.1 \% \mathrm{OM}$, and $14.8 \%$ IPD. For NVT20, proportions were $38.4 \%$ in carriage in non-LRI, $34.4 \%$ carriage in LRI, $34.4 \%$ for conjunctivitis, $20.9 \%$ OM, and $13.4 \%$ IPD.

\section{Proportion Dynamics of VT13, VT20-13, and NVT20 Comparing Late PCV13 Period with Early PCV Period}

The proportions of VT13 of all isolates declined significantly by $73 \%-86 \%$ in all groups when comparing late-PCV13 period with early-PCV period. During the late-PCV13 period, VT13 were identified in only $9 \%-14 \%$ of all pneumococcal isolates in the various groups (Figures 1, 2).
A

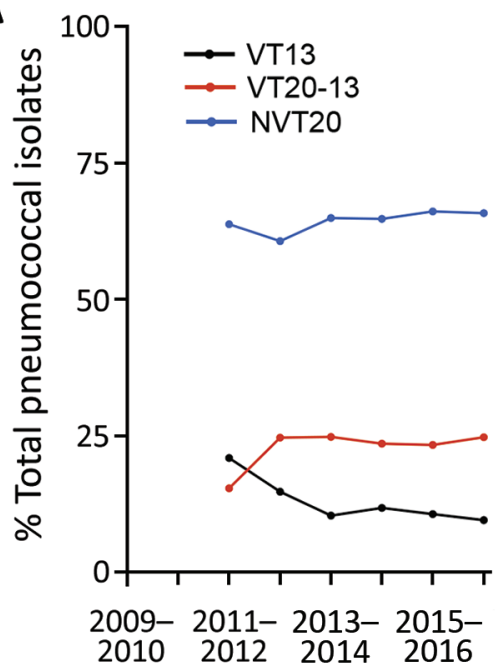

B

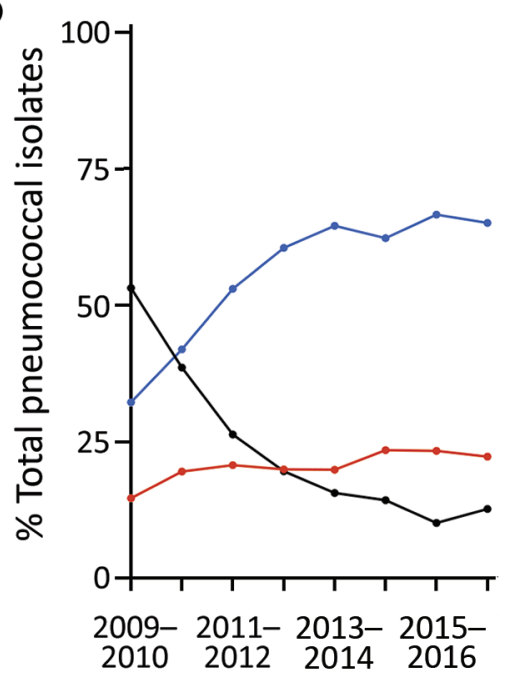

E

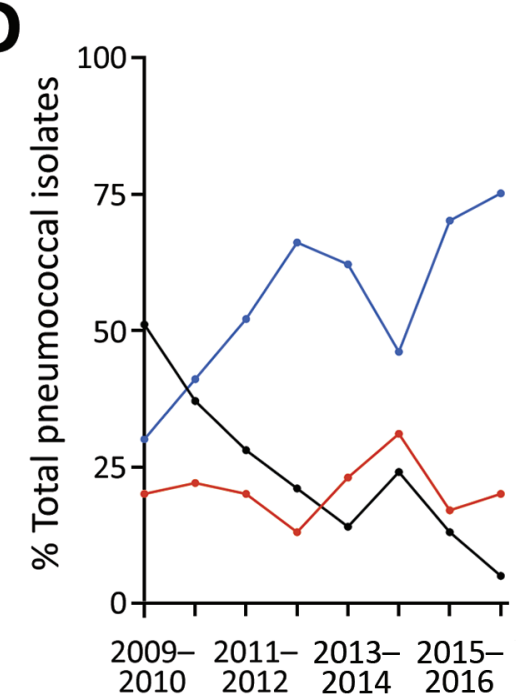

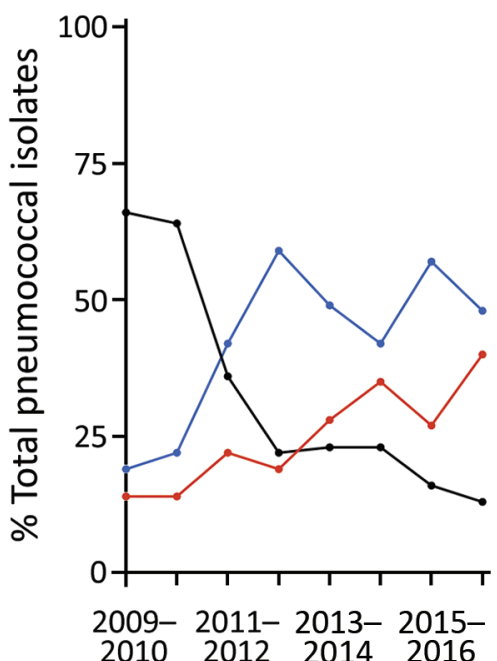

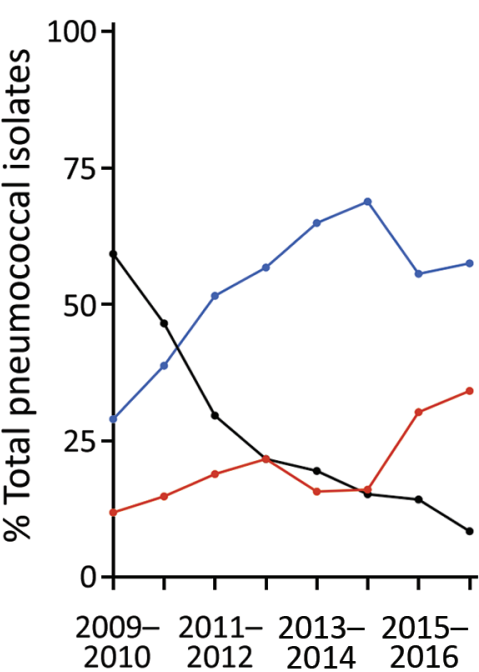

$\mathbf{F}$

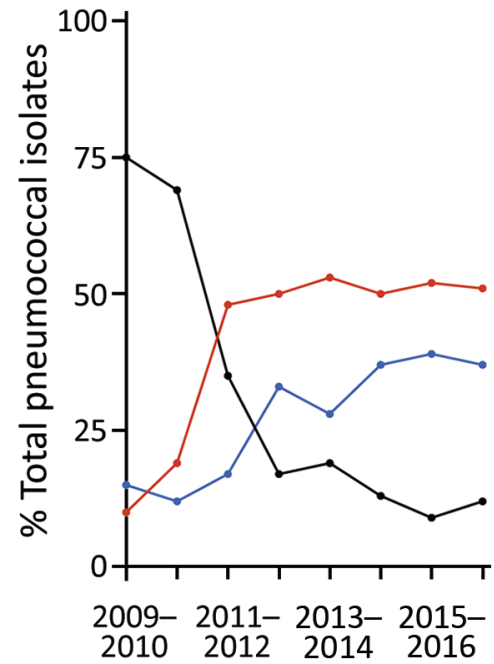

Figure 1. Postvaccine dynamics of pneumococcal conjugate vaccines in children $<24$ months of age, Israel, July 2009-June 2017. A) Healthy children; B) children with non-LRTIs; C) children with lower respiratory infections; D) children with conjunctivitis; E) children with otitis media (isolates from middle ear fluid were tested); and F) children with invasive pneumococcal disease (isolates from blood and cerebrospinal fluid were tested). Data show VT13, VT20-13, and NVT20 as the proportion of each serotype of all pneumococcal isolates. $p<0.05$ for all comparisons comparing 2015-2017 versus 2009-2011, except for VT20-13 in conjunctivitis. LRTI, lower respiratory tract infection; NVT, nonvaccine serotype; VT, vaccine serotype. 

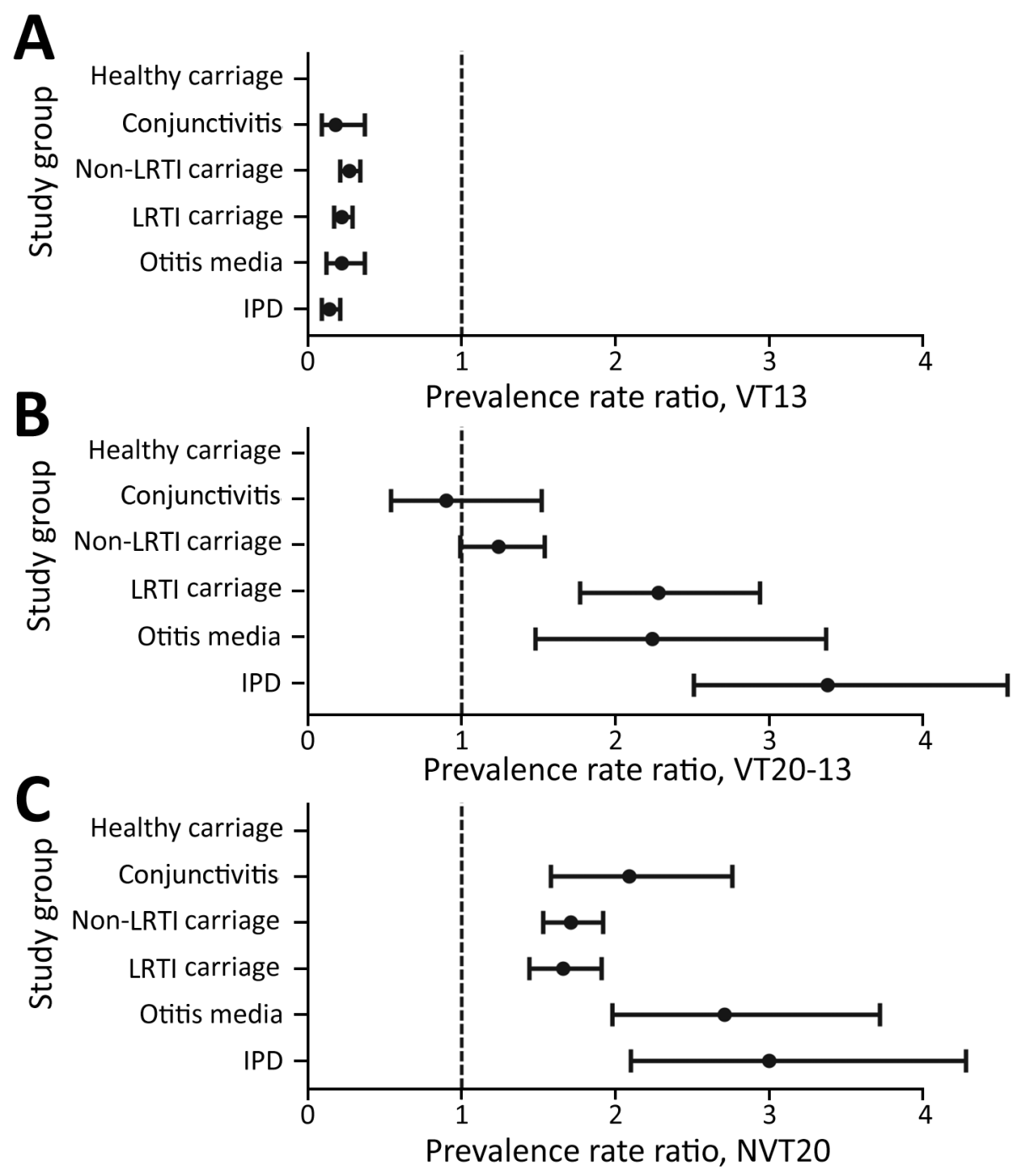

Figure 2. Prevalence rate ratios of pneumococcal VT13, VT20-13, and NVT20 of all pneumococcal isolates in carriage, conjunctivitis, $\mathrm{OM}$, and IPD in children $<24$ months of age, Israel, comparing the late PCV13 period (2015-2017) to the early PCV period (20092011). The comparison could not be done for carriage in healthy children due to the nonavailability of data for the early PCV period. $p<0.05$ for all comparisons. Error bars indicate $95 \% \mathrm{Cls}$. IPD, invasive pneumococcal disease; LRTI, lower respiratory tract infection; NVT, nonvaccine serotype; OM, otitis media; PCV, pneumococcal conjugate vaccine; PCV13, 13-valent PCV; $\mathrm{VT}$, vaccine serotype.
Proportions of VT20-13 did not increase significantly in conjunctivitis and carriage in non-LRI illness (Table 1; Figure 2). In contrast, we observed a significant increase of carriage in LRI, OM, and IPD ( $p<0.05$ by test for trend in proportion for all groups). During the last study year, the proportions of VT20-13 of all identified pneumococcal isolates were $20 \%-25 \%$ in carriage in healthy children, carriage in non-LRTI, and conjunctivitis; 33\% in carriage in LRTI; $40 \%$ in carriage in OM; and 51\% in IPD. The fraction of VT20-13 became the leading one in IPD during the late-PCV13 period. The proportion rate ratios (20152017 vs. 2009-2011) for carriage in LRI, OM, and IPD were similar, whereas ratios for all 3 were significantly higher than those for conjunctivitis and carriage in non-LRTI disease.

Proportions of non-NVT20 increased in all groups, ranging from $66 \%-200 \%$ increase. The increase in IPD was significantly higher than in carriage in non-LRI and carriage in LRTI, but other increases did not differ significantly (Figure 2).

\section{VT20-13/NVT20 Ratio}

To assess the relative role of VT20-13 among all NVT, we calculated the ratio between VT20-13 and NVT20. In the early PCV period, VT20-13/NVT20 ratio was the lowest for carriage in LRTI (0.39) followed by carriage in non-LRTI (0.46), conjunctivitis (0.60), OM (0.68), and IPD (1.19) (Table 2). In the late-PCV13 period (2015-2017), VT20-13/NVT20 ratio was the lowest in conjunctivitis (0.26), followed by carriage in healthy children (0.37) and carriage in non-LRTI diseases (0.35) (Figure 3). The ratios for OM and carriage in LRTI were significantly higher than for the conjunctivitis, carriage in non-LRTI, and carriage in healthy children groups. The highest ratio was observed for IPD (1.40), significantly higher than all other outcomes. 
Table 2. Proportions of VT20-13/NVT20 ratios in children age <24 mo, Israel, 2009-2011 and 2015-2017*

\begin{tabular}{|c|c|c|c|c|c|c|c|}
\hline \multirow[b]{2}{*}{ Clinical characteristic } & \multicolumn{3}{|c|}{ 2009-2011 } & \multicolumn{3}{|c|}{ 2015-2017 } & \multirow{2}{*}{$\begin{array}{c}\text { 2015-2017 vs. } \\
2009-2011 \\
\text { VT20-13/NVT20 } \\
\text { ratio }(95 \% \mathrm{Cl})\end{array}$} \\
\hline & $\begin{array}{c}\text { VT20-13, } \\
\%\end{array}$ & $\begin{array}{l}\text { NVT20, } \\
\%\end{array}$ & $\begin{array}{l}\text { VT20-13/NVT20 } \\
\text { ratio }(95 \% \mathrm{CI})\end{array}$ & $\begin{array}{c}\text { VT20-13, } \\
\%\end{array}$ & $\begin{array}{l}\text { NVT20, } \\
\%\end{array}$ & $\begin{array}{l}\text { VT20-13/NVT20 } \\
\text { ratio }(95 \% \mathrm{Cl})\end{array}$ & \\
\hline $\begin{array}{l}\text { Carriage in healthy } \\
\text { children }\end{array}$ & NA & NA & NA & 24 & 66 & $0.37(0.33-0.41)$ & NA \\
\hline Carriage in non-LRTI & 18 & 38 & $0.46(0.38-0.56)$ & 23 & 66 & $0.35(0.30-0.40)$ & $0.80(0.65-0.98)$ \\
\hline Conjunctivitis & 20 & 34 & $0.60(0.39-0.91)$ & 19 & 72 & $0.26(0.17-0.40)$ & $0.57(0.35-0.94)$ \\
\hline Carriage in LRTI & 13 & 34 & $0.39(0.30-0.50)$ & 32 & 57 & $0.56(0.48-0.65)$ & $1.26(1.00-1.60)$ \\
\hline OM & 14 & 21 & $0.68(0.49-0.94)$ & 34 & 52 & $0.60(0.44-0.81)$ & $0.91(0.66-1.25)$ \\
\hline IPD & 15 & 13 & $1.19(0.79-1.80)$ & 52 & 38 & $1.40(1.12-1.73)$ & $1.05(0.84-1.31)$ \\
\hline
\end{tabular}

\section{Serotype-Specific VT20-13 Dynamics}

Of all 2,126 VT20-13 isolates, serotype 15B/C was the most common $(40.7 \%)$, followed by serotypes $11 \mathrm{~A}$ $(16.9 \%), 12 \mathrm{~F}(12.8 \%), 33 \mathrm{~F}(10.4 \%), 10 \mathrm{~A}(9.6 \%), 22 \mathrm{~F}$ $(7.6 \%)$, and $8(2.0 \%)$. (Figure 4; Appendix Table 2). Of note, serotype $15 \mathrm{~B} / \mathrm{C}$ was the most common VT20-13 serotype in all clinical syndromes; it constituted $37 \%-$ $49 \%$ of all VT20-13 in each group, except for IPD, in which serotype $12 \mathrm{~F}$ was the most common $(50 \%$ of all VT20-13).

\section{Antimicrobial Nonsusceptibility Rates among VT20-13 and NVT20 during the Late PCV13 Period}

Antibiotic nonsusceptibility was higher among VT2013 as a group than NVT20 for erythromycin, tetracycline, trimethoprim-sulfamethoxazole, clindamycin, and for MDR (Table 3, https://wwwnc.cdc.gov/ EID/article/27/1/20-1178-T3.htm; Figure 5). Similarly, in IPD, proportions of isolates with penicillin nonsusceptibility (MIC $\geq 0.1 \mu \mathrm{g} / \mathrm{mL}$ ) were higher in VT20-13 compared with NVT20. In contrast, penicillin nonsusceptibility (MIC $\geq 0.1 \mu \mathrm{g} / \mathrm{mL}$ ) was more prevalent in NVT20 than in VT20-13 in the all outcomes group; for penicillin nonsusceptibility with

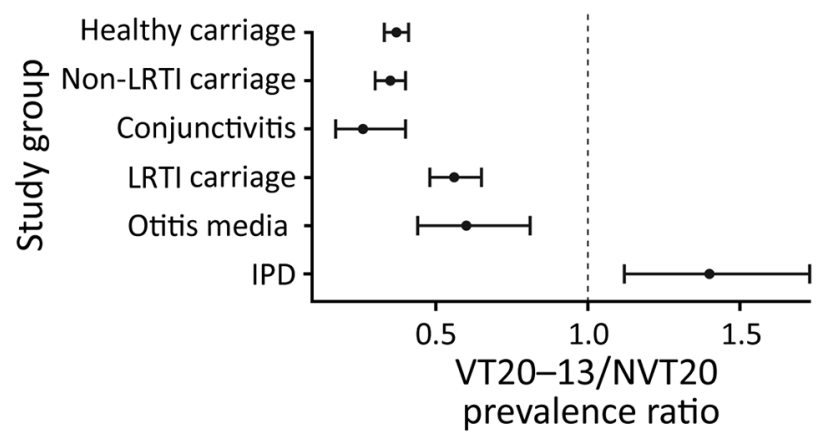

Figure 3. Ratio of prevalence of pneumococcal VT20-13/NVT20 ratio in children $<24$ months of age, Israel, during the late PCV13 period (2015-2017). Error bars represent 95\% CI. IPD, invasive pneumococcal disease; LRTI, lower respiratory tract infection; NVT, nonvaccine serotype; PCV, pneumococcal conjugate vaccine; PCV13, 13-valent PCV; VT, vaccine serotype.
MIC $\geq 1.0 \mu \mathrm{g} / \mathrm{mL}$, we observed these trends in carriage in healthy children, carriage in non-LRTI, and carriage in LRTI.

\section{Discussion}

Widespread implementation of PCVs has universally resulted in complete or near-complete nasopharyngeal colonization replacement of VT by NVT $(9,18,19)$. This, in turn, resulted in increased disease by NVT to a variable extent, depending mainly on the disease entity (i.e., mucosal or invasive diseases) and environmental and host factors $(3,4)$. The currently licensed PCVs contain $\leq 13$ serotypes, and the remaining $>85$ serotypes constitute potential replacing serotypes. However, not all serotypes are equally capable of progressing from colonization to disease, and not all are equally successful colonizers, resulting in selection of specific serotypes that will eventually be frequently associated with disease. Furthermore, the most successful colonizers will likely increasingly become nonsusceptible to the commonly used antimicrobial drugs (20-22). Therefore, although invasive and mucosal pneumococcal disease are invariably reduced in children after PCV implementation, the remaining potential disease may be related disproportionally to a relatively small fraction of the remaining serotypes. In this study, we have shown that as a group, the 7 serotypes selected to be included in PCV20 are indeed disproportionally associated with disease and were frequently more drug-nonsusceptible than the other remaining NVT20. Although VT20-13/NVT20 ratios declined throughout the study for carriage in non-LRTI and conjunctivitis, these ratios increased or remained stable for carriage in LRTI, OM, and IPD. These trends point to the high disease potential of VT20-13 compared with NVT20 for IPD (in which VT20-13 caused $>50 \%$ of all episodes in the last study period), and possibly for certain pneumococcal mucosal diseases such as $\mathrm{OM}$ and LRTI (in which VT20-13/NVT20 ratio increased, but NVT20 caused $>50 \%$ of all episodes). We believe that 
these trends were driven by the vaccine-induced neardisappearance of the generally invasive PCV13 serotypes and the gradual differential replacement of those serotypes by NVT in different clinical syndromes, according to the specific NVT disease potential.

Several of the 7 additional PCV20 serotypes have been previously shown to have a high disease potential for IPD and other pneumococcal diseases $(4,5,23-26)$. Nevertheless, all 7 serotypes were recognized as causing diseases and therefore were included in the 23-valent polysaccharide vaccine (PPV23), commonly used for the prevention of IPD in adults and in children $\geq 2$ years of age with immunodeficiency and certain chronic medical conditions $(4,27,28)$. Specifically, serotype $12 \mathrm{~F}$ emerged as a major serotype in multiple countries, causing IPD and pneumonia $(5,10,22-24,29,30)$. Its expansion was multiclonal, excluding a single hyperinvasive clone outbreak (26). This pattern bears similarities with the expansion of serotype 7F that occurred following PCV7 implementation; both serotypes were not expected to increase because they were frequently associated with low carriage. Serotype 8 was relatively

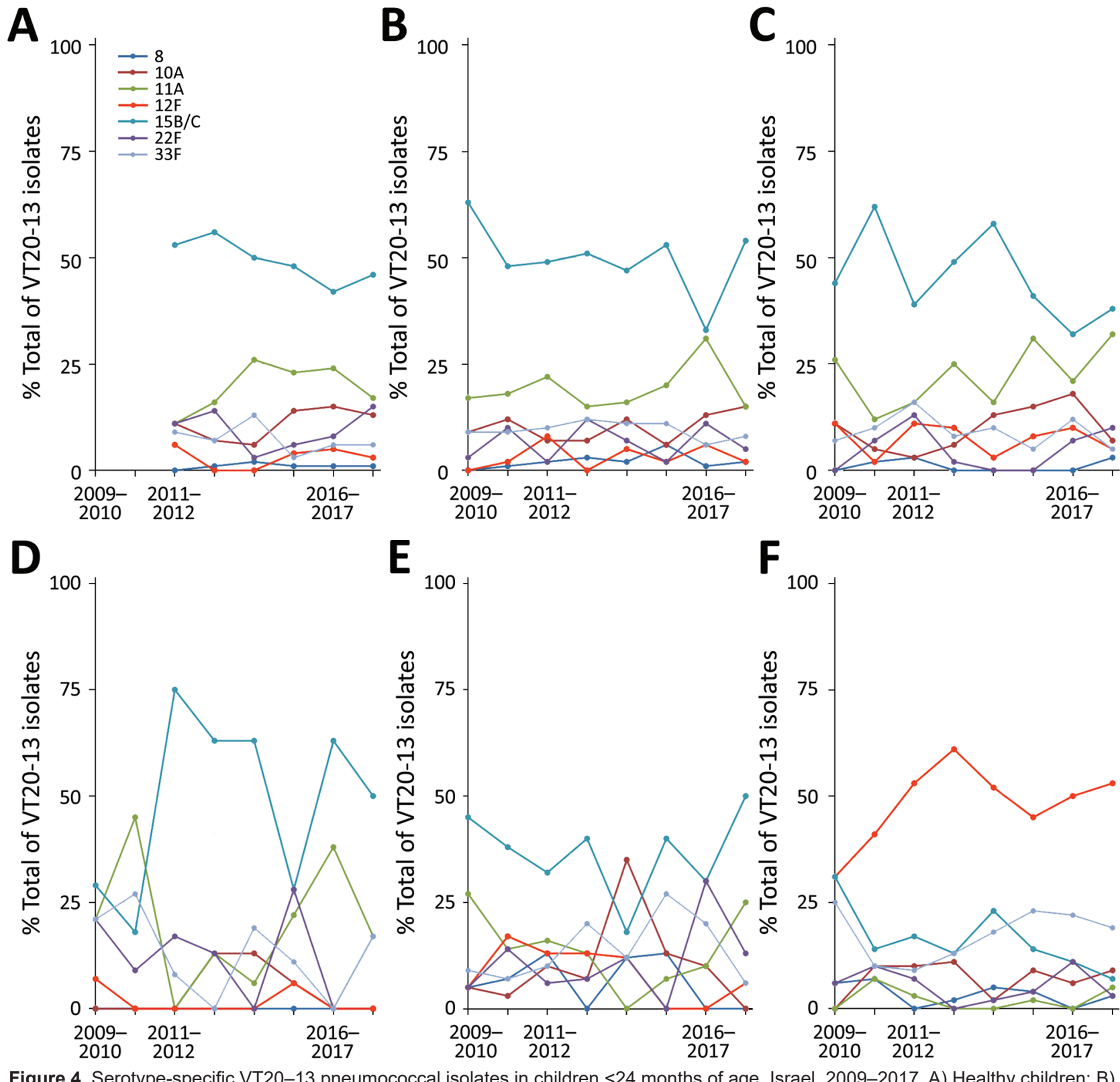

Figure 4. Serotype-specific VT20-13 pneumococcal isolates in children <24 months of age, Israel, 2009-2017. A) Healthy children; B) children with non-lower respiratory tract infections; C) children with lower respiratory tract infections; D) children with conjunctivitis; E) children with otitis media (isolates from middle ear fluid were tested); and F) children with invasive pneumococcal disease (isolates from blood and cerebrospinal fluid were tested). VT, vaccine serotype. 

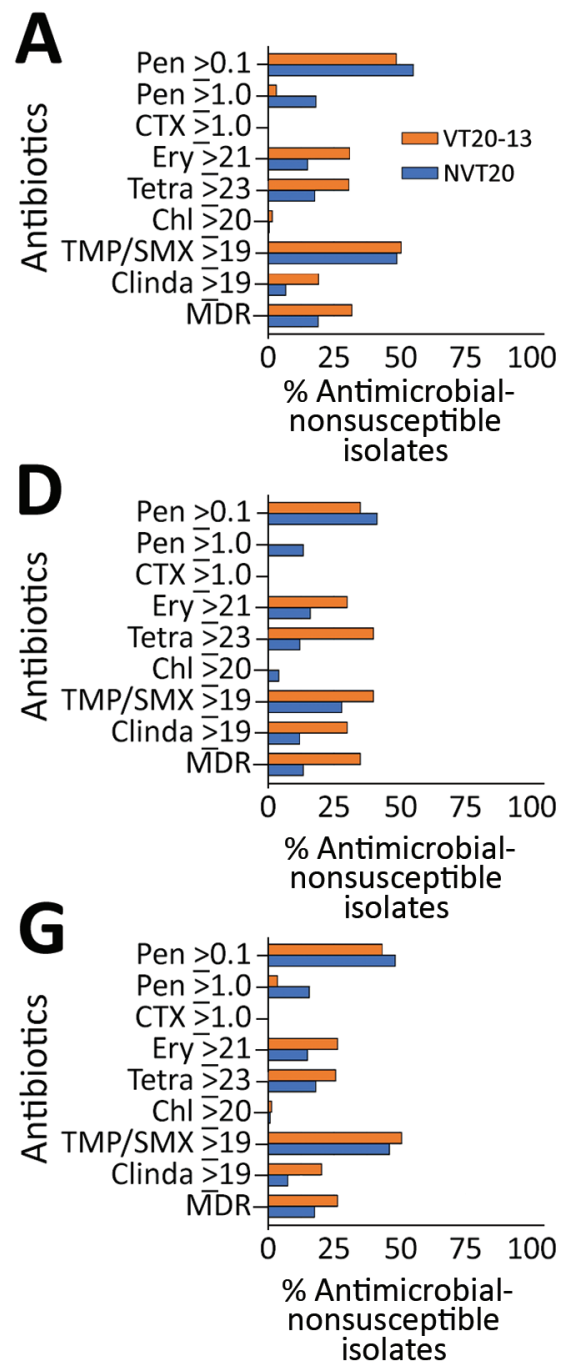
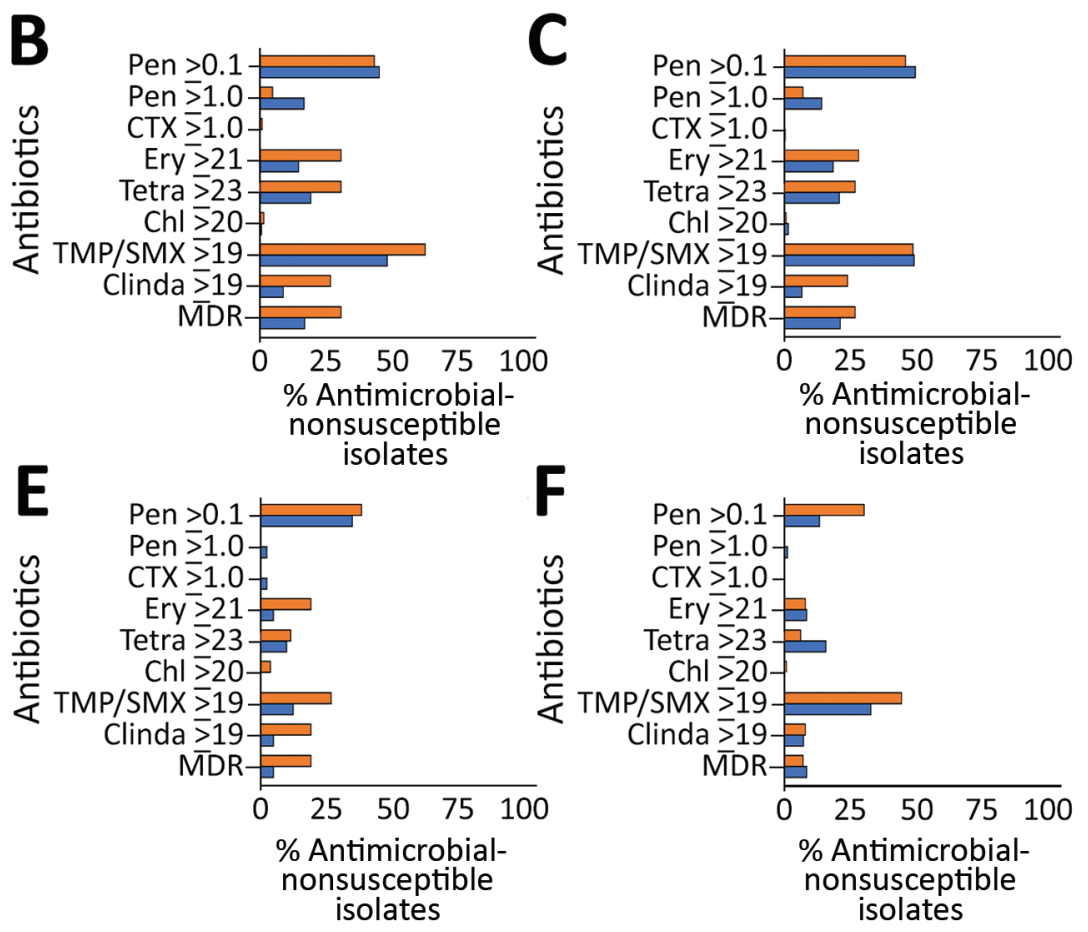

Figure 5. Proportion of antimicrobial drug-nonsusceptible isolates of all pneumococcal isolates in VT20-13 versus non-VT20 pneumococcal isolates in the late PCV13 period (2015-2017) in children <24 months of age in southern Israel. A) Healthy children; B) children with non-lower respiratory tract infections; C) children with lower respiratory tract infections; D) children with conjunctivitis; E) children with otitis media (isolates from middle ear fluid were tested); F) children with invasive pneumococcal disease; and G) all children. Chl, chloramphenicol; Clinda, clindamycin; CTX, ceftriaxone; Ery, erythromycin; MDR, multidrug resistance; PCV, pneumococcal conjugate vaccine; PCV13, 13-valent PCV; Pen, penicillin; Tetra, tetracycline; TMP/ SMX, trimethoprim and sulphametoxazole; VT, vaccine serotype. uncommon in the current study but has emerged as one of the most prominent replacing serotypes in IPD in adults after PCV implementation, including in Israel $(23-25,31)$. However, reports of this serotype in children are also emerging (26). Serotypes $22 \mathrm{~F}$ and $33 \mathrm{~F}$, which are also included in an experimental 15-valent PCV currently in an advanced stage of clinical studies $(11,32,33)$, were recognized as important serotypes in IPD and pneumonia $(11,24,25,34)$. In the United States, proportions of IPD caused by serotype $22 \mathrm{~F}$ were $11 \%$ in children $<5$ years and $13 \%$ in adults $\geq 18$ years, whereas serotype $33 \mathrm{~F}$ caused $10 \%$ of residual IPD cases in children $<5$ years and $5 \%$ in adults $\geq 18$ years $(11,32)$. In addition, serotypes 10A, 11A, and 15B/C, considered to have lower disease potential than serotypes $12 \mathrm{~F}, 8$, $22 \mathrm{~F}$, and $33 \mathrm{~F}$, have been reported frequently following PCV implementation worldwide (5,10,23-25).

Despite the importance of VT20 as a group, several NVT20 have been shown to be able to have the potential to cause large outbreaks (i.e., pneumococcal serotype 2 responsible for large IPD outbreaks in Israel) (35) or reported as emerging IPD-causing serotypes (i.e., serotype $24 \mathrm{~F})(5,6,22)$. Others, considered less invasive NVT20 serotypes have been also reported in several sites (i.e., serotypes 15A and 16F) (36-38).

This study adds important information not only on the proportions of specific serotypes causing IPD in the PCV13 era, but also with regard to the proportions of specific serotypes in carriage in healthy children versus children with IPD and mucosal diseases. These data are relevant because carriage during disease probably reflects relative disease potential in the various clinical entities. We found that both the rates of incidence and the relative proportion of VT20-13 seen in IPD, OM, and carriage in LRTI requiring chest radiography were significantly higher than those in conjunctivitis and in carriage in non-LRTI not requiring chest radiography. Specifically, in IPD, serotype 
$12 \mathrm{~F}$ emerged as the most common serotype causing IPD in children $<2$ years of age, with $\approx 25 \%$ of all IPD caused by this serotype in recent years. Thus, the addition of serotype 12F coverage in PCV20 is expected to further decrease overall IPD rates in this population. Similarly, serotype 15B/C was the most common serotype in pneumococcal carriage and mucosal diseases $(\approx 10 \%$ of all pneumococcal isolates).

We found that antimicrobial nonsusceptibility was significantly more frequent among VT20-13 than among NVT20; other studies have also shown such increases among PCV20-13 serotypes $(21,22,39,40)$. In the pre-PCV era, most nonsusceptible strains belonged to serotypes included in PCV7/PCV13. Although nonsusceptibility is still higher among those strains or even increasing, the overall nonsusceptibility has been reduced in countries which successfully implemented PCVs $(21,22,39)$. However, high selective pressure continues to occur with excessive antibiotic consumption, especially in dominating carriage serotypes. The most successful replacing serotypes are now those frequently exposed to antimicrobial drugs during carriage, resulting in increased antimicrobial nonsusceptibility compared with the less successful colonizers often found among NVT20 $(21,22,39,40)$. Of interest, isolates causing noninvasive disease tend to have higher rates of antimicrobial resistance than those causing IPD (21), similar to the observations in our study.

The main strengths of our study include relatively long-term surveillance duration, large number of episodes, and the ability to assess multiple clinical outcomes in the same populations. Nonetheless, our study has several limitations. First, we did not have data on the pre-PCV period. However, we still could follow the dynamics of the non-PCV7/PCV13 serotypes because the data from the first year of implementation are included. Second, with regard to carriage in healthy children, data are only available from 2011-2012. However, the similarity of the proportion rates for 2010-2011 when carriage in healthy children is compared with that of children with non-LRTI and children with conjunctivitis (the most superficial mucosal disease, potentially explaining the higher proportion of noninvasive serotypes in the disease compared with other diseases) lends support to the suggestion that there are similarities between these groups and healthy children during the entire study period. Third, our data derive from multiple studies in the setting of a single country. However, as discussed above, similarities with other reports from other countries suggest that our conclusion may be generalized, at least to some extent.

In conclusion, S. pneumoniae VT20-13 are disproportionally associated with IPD, OM, and carriage in
LRTI, compared with the other remaining NVT20, suggesting higher disease potential for these diseases than NVT20. In addition, the VT20-13 serotypes were more often nonsusceptible to various antimicrobial drugs than the NVT20 group. These findings suggest that PCV20 introduction may result in substantial decrease in the rates of IPD, OM, and possibly LRTI, as well as antimicrobial nonsusceptibility in children.

This work was supported in part by a grant from Pfizer (grant no. 0887X1-4603). The funders had no role in study design, data collection and interpretation, or the decision to submit the work for publication.

S. B.-S. has received speakers' fees and a grant from Pfizer, has been a scientific consultant for Merck Sharp \& Dohme, and has served as a speaker for GlaxoSmithKline and Merck Sharp \& Dohme. D.G. has received grants from Merck Sharp \& Dohme; has been a scientific consultant for GlaxoSmithKline, Merck Sharp \& Dohme, and Pfizer; and has served as a speaker for GlaxoSmithKline, Merck Sharp \& Dohme, and Pfizer. R.D. has received grants and research support from Pfizer, Merck Sharp \& Dohme, and Medimmune; has been a scientific consultant for Pfizer, MeMed, Merck Sharp \& Dohme, and Biondvax; had served on advisory boards of Pfizer, Merck Sharp \& Dohme, and Biondvax; and has served as a speaker for Pfizer.

\section{About the Author}

Dr. Ben-Shimol is a specialist in pediatric infectious diseases at the Pediatric Infectious Disease Unit of the Soroka University Medical Center and an associate professor at the Faculty of Health Sciences of the Ben-Gurion University of the Negev, Beer-Sheva, Israel. His research interests are pneumococcal infectious diseases, pneumococcal vaccines, zoonotic diseases, and parasitology.

\section{References}

1. O'Brien KL, Wolfson LJ, Watt JP, Henkle E, Deloria-Knoll M, McCall N, et al.; Hib and Pneumococcal Global Burden of Disease Study Team. Burden of disease caused by Streptococcus pneumoniae in children younger than 5 years: global estimates. Lancet. 2009;374:893-902. https://doi.org/ 10.1016/S0140-6736(09)61204-6

2. Wahl B, O'Brien KL, Greenbaum A, Majumder A, Liu L, Chu Y, et al. Burden of Streptococcus pneumoniae and Haemophilus influenzae type $\mathrm{b}$ disease in children in the era of conjugate vaccines: global, regional, and national estimates for 2000-15. Lancet Glob Health. 2018;6:e744-57. https://doi.org/10.1016/S2214-109X(18)30247-X

3. Jedrzejas MJ. Pneumococcal virulence factors: structure and function. Microbiol Mol Biol Rev. 2001;65:187-207. https:/ / doi.org/10.1128/MMBR.65.2.187-207.2001

4. Bogaert D, Hermans PWM, Adrian PV, Rümke HC, de Groot R. Pneumococcal vaccines: an update on current strategies. Vaccine. 2004;22:2209-20. https://doi.org/ 10.1016/j.vaccine.2003.11.038 
5. Balsells E, Guillot L, Nair H, Kyaw MH. Serotype distribution of Streptococcus pneumoniae causing invasive disease in children in the post-PCV era: A systematic review and metaanalysis. PLoS One. 2017;12:e0177113. https:/ / doi.org/ 10.1371/journal.pone.0177113

6. Ben-Shimol S, Dagan R, Givon-Lavi N, Avital D, Bar-Ziv J, Greenberg D. Use of chest radiography examination as a probe for pneumococcal conjugate vaccine impact on lower respiratory tract infections in young children. Clin Infect Dis. 2019;71:177-87. 10.1093/cid/ciz768 https:/ / doi.org/10.1093/cid/ciz768

7. Ben-Shimol S, Givon-Lavi N, Leibovitz E, Raiz S, Greenberg D, Dagan R. Near-elimination of otitis media caused by 13-valent pneumococcal conjugate vaccine (PCV) serotypes in southern Israel shortly after sequential introduction of 7-valent/13-valent PCV. Clin Infect Dis. 2014;59:1724-32. https://doi.org/10.1093/cid/ciu683

8. de Oliveira LH, Camacho LAB, Coutinho ESF, MartinezSilveira MS, Carvalho AF, Ruiz-Matus C, et al. Impact and effectiveness of 10 and 13-valent pneumococcal conjugate vaccines on hospitalization and mortality in children aged less than 5 years in Latin American countries: a systematic review. PLoS One. 2016;11:e0166736. https:/ / doi.org/ 10.1371/journal.pone.0166736

9. Ben-Shimol S, Givon-Lavi N, Greenberg D, Dagan R. Pneumococcal nasopharyngeal carriage in children $<5$ years of age visiting the pediatric emergency room in relation to PCV7 and PCV13 introduction in southern Israel. Hum Vaccin Immunother. 2016;12:268-76. https:/ / doi.org/10.1080/ 21645515.2015.1095414

10. Thompson A, Lamberth E, Severs J, Scully I, Tarabar S, Ginis J, et al. Phase 1 trial of a 20-valent pneumococcal conjugate vaccine in healthy adults. Vaccine. 2019;37:6201-7. https:/ / doi.org/10.1016/j.vaccine.2019.08.048

11. Rupp R, Hurley D, Grayson S, Li J, Nolan K, McFetridge RD, et al. A dose ranging study of 2 different formulations of 15-valent pneumococcal conjugate vaccine (PCV15) in healthy infants. Hum Vaccin Immunother. 2019;15:549-59. https:// doi.org/10.1080/21645515.2019.1568159

12. Ben-Shimol S, Givon-Lavi N, Leibovitz E, Greenberg D, Dagan R. Studying PCV impact on clinical presentation of otitis media helps to understand its pathogenesis. Vaccine. 2019;37:1-6. https://doi.org/10.1016/j.vaccine.2018.11.054

13. Ben-Shimol S, Givon-Lavi N, Leibovitz E, Raiz S, Greenberg D, Dagan R. Impact of widespread introduction of pneumococcal conjugate vaccines on pneumococcal and nonpneumococcal otitis media. Clin Infect Dis. 2016;63:611-8. https://doi.org/10.1093/cid/ciw347

14. Ben-Shimol S, Greenberg D, Givon-Lavi N, Schlesinger Y, Somekh E, Aviner S, et al. Early impact of sequential introduction of 7-valent and 13-valent pneumococcal conjugate vaccine on IPD in Israeli children $<5$ years: An active prospective nationwide surveillance. Vaccine. 2014;32:3452-9.10

15. Buznach N, Dagan R, Greenberg D. Clinical and bacterial characteristics of acute bacterial conjunctivitis in children in the antibiotic resistance era. Pediatr Infect Dis J. 2005;24:8238. https:/ / doi.org/10.1097/01.inf.0000178066.24569.98

16. Ben-Shimol S, Greenberg D, Givon-Lavi N, Elias N, Glikman D, Rubinstein U, et al.; Israeli Bacteremia and Meningitis Active Surveillance Group. Rapid reduction in invasive pneumococcal disease after introduction of PCV7 into the National Immunization Plan in Israel. Vaccine. 2012;30:6600-7. https://doi.org/10.1016/j.vaccine.2012.08.012

17. Clinical and Laboratory Standards Institute. Performance standards for antimicrobial susceptibility testing: 28 th edition informational supplement (M100-S28). Wayne (PA): The Institute; 2018.

18. Weinberger DM, Malley R, Lipsitch M. Serotype replacement in disease after pneumococcal vaccination. Lancet. 2011;378:1962-73. https:/ / doi.org/10.1016/ S0140-6736 (10)62225-8

19. Usuf E, Bottomley C, Bojang E, Cox I, Bojang A, Gladstone R, et al. Persistence of nasopharyngeal pneumococcal vaccine serotypes and increase of nonvaccine serotypes among vaccinated infants and their mothers 5 years after introduction of pneumococcal conjugate vaccine 13 in The Gambia. Clin Infect Dis. 2019;68:1512-21. https://doi.org/10.1093/cid/ciy726

20. Danino D, Givon-Lavi N, Ben-Shimol S, Greenberg D, Dagan R. Understanding the evolution of antibioticnonsusceptible pneumococcal nasopharyngeal colonization following pneumococcal conjugate vaccine implementation in young children. Clin Infect Dis. 2019;69:648-56. https://doi.org/10.1093/cid/ciy926

21. Richter SS, Diekema DJ, Heilmann KP, Dohrn CL, Riahi F, Doern GV. Changes in pneumococcal serotypes and antimicrobial resistance after introduction of the 13-valent conjugate vaccine in the United States. Antimicrob Agents Chemother. 2014;58:6484-9. https:// doi.org/10.1128/AAC.03344-14

22. Varon E, Cohen R, Béchet S, Doit C, Levy C. Invasive disease potential of pneumococci before and after the 13-valent pneumococcal conjugate vaccine implementation in children. Vaccine. 2015;33:6178-85. https:/ / doi.org/10.1016/j.vaccine.2015.10.015

23. Amin-Chowdhury Z, Iyanger N, Ramsay ME, Ladhani SN. Outbreaks of severe pneumococcal disease in closed settings in the conjugate vaccines era, 2010-2018: A systematic review to inform national guidance in the UK. J Infect. 2019;79:495502. https:/ / doi.org/10.1016/j.jinf.2019.10.009

24. Pick H, Daniel P, Rodrigo C, Bewick T, Ashton D, Lawrence H, et al. Pneumococcal serotype trends, surveillance and risk factors in UK adult pneumonia, 2013-18. Thorax. 2020;75:38-49. https://doi.org/10.1136/ thoraxjnl-2019-213725

25. Levy C, Ouldali N, Caeymaex L, Angoulvant F, Varon E, Cohen R. Diversity of serotype replacement after pneumococcal conjugate vaccine implementation in Europe. J Pediatr. 2019;213:252-253.e3. https:/ / doi.org/10.1016/ j.jpeds.2019.07.057

26. Lo SW, Gladstone RA, van Tonder AJ, Lees JA, du Plessis M, Benisty R, et al.; Global Pneumococcal Sequencing Consortium. Pneumococcal lineages associated with serotype replacement and antibiotic resistance in childhood invasive pneumococcal disease in the post-PCV13 era: an international whole-genome sequencing study. Lancet Infect Dis. 2019;19:759-69. https:/ / doi.org/10.1016/S1473-3099(19)30297-X

27. Hausdorff WP, Feikin DR, Klugman KP. Epidemiological differences among pneumococcal serotypes. Lancet Infect Dis. 2005;5:83-93. https://doi.org/10.1016/S1473-3099(05)70083-9

28. Djennad A, Ramsay ME, Pebody R, Fry NK, Sheppard C, Ladhani SN, et al. Effectiveness of 23-valent polysaccharide pneumococcal vaccine and changes in invasive pneumococcal disease incidence from 2000 to 2017 in those aged 65 and over in England and Wales. EClinicalMedicine. 2018;6:42-50. https://doi.org/10.1016/j.eclinm.2018.12.007

29. Rokney A, Ben-Shimol S, Korenman Z, Porat N, Gorodnitzky Z, Givon-Lavi N, et al. Emergence of Streptococcus pneumoniae serotype $12 \mathrm{~F}$ after sequential introduction of 7- and 13-valent vaccines, Israel. Emerg Infect Dis. 2018;24:453-61. https://doi.org/10.3201/eid2403.170769

30. Cohen R, Levy C, Bonnet E, Thollot F, Boucherat M, Fritzell B, et al. Risk factors for serotype 19A carriage after 
introduction of 7-valent pneumococcal vaccination. BMC Infect Dis. 2011;11:95. https:/ / doi.org/10.1186/ 1471-2334-11-95

31. Regev-Yochay G, Katzir M, Strahilevitz J, Rahav G, Finn T, Miron D, et al.; IAIPD group. The herd effects of infant PCV7/PCV13 sequential implementation on adult invasive pneumococcal disease, six years post implementation; a nationwide study in Israel. Vaccine. 2017; 35:2449-56. https:// doi.org/10.1016/j.vaccine.2017.03.031

32. Moore MR, Link-Gelles R, Schaffner W, Lynfield R, Lexau C, Bennett NM, et al. Effect of use of 13-valent pneumococcal conjugate vaccine in children on invasive pneumococcal disease in children and adults in the USA: analysis of multisite, population-based surveillance. Lancet Infect Dis. 2015;15:301-9. https:/ / doi.org/10.1016/ S1473-3099(14)71081-3

33. Pilishvili T, Lexau C, Farley MM, Hadler J, Harrison LH, Bennett NM, et al.; Active Bacterial Core Surveillance/ Emerging Infections Program Network. Sustained reductions in invasive pneumococcal disease in the era of conjugate vaccine. J Infect Dis. 2010;201:32-41. https://doi.org/10.1086/648593

34. van Hoek AJ, Andrews N, Waight PA, George R, Miller E. Effect of serotype on focus and mortality of invasive pneumococcal disease: coverage of different vaccines and insight into non-vaccine serotypes. PLoS One. 2012;7:e39150. https://doi.org/10.1371/journal.pone.0039150

35. Dagan R. Ben-Shimol S, Benisty R, Regev- Yochay G, Ron M, Givon-Lavi N, et al. A nationwide outbreak of invasive pneumococcal disease (IPD) caused by a novel Streptococcus pneumoniae serotype 2 (SP2) clone in the PCV13 era, in Israel. Abstract 1888. In: Abstracts of IDweek 2019; October 2-6, 2019; Washington, DC, USA. Alexandria (VA): Infectious Diseases Society of America; 2019.

36. Nakano S, Fujisawa T, Ito Y, Chang B, Matsumura Y, Yamamoto $\mathrm{M}$, et al. Whole-genome sequencing analysis of multidrug-resistant serotype 15A Streptococcus pneumoniae in Japan and the emergence of a highly resistant serotype 15A-ST9084 clone. Antimicrob Agents Chemother. 2019;63:e02579-18. https:// doi.org/10.1128/AAC.02579-18

37. Neves FPG, Cardoso NT, Cardoso CAA, Teixeira LM, Riley LW. Direct effect of the 13-valent pneumococcal conjugate vaccine use on pneumococcal colonization among children in Brazil. Vaccine. 2019;37:5265-9. https://doi.org/10.1016/ j.vaccine.2019.07.056

38. Dayie NTKD, Tettey EY, Newman MJ, Bannerman E, Donkor ES, Labi A-K, et al. Pneumococcal carriage among children under five in Accra, Ghana, five years after the introduction of pneumococcal conjugate vaccine. BMC Pediatr. 2019;19:316. https://doi.org/10.1186/ s12887-019-1690-5

39. Cohen R, Biscardi S, Levy C. The multifaceted impact of pneumococcal conjugate vaccine implementation in children in France between 2001 to 2014. Hum Vaccin Immunother. 2016;12:277-84. https:/ / doi.org/10.1080/21645 515.2015.1116654

40. Tomczyk S, Lynfield R, Schaffner W, Reingold A, Miller L, Petit $S$, et al. Prevention of antibiotic-nonsusceptible invasive pneumococcal disease with the 13-valent pneumococcal conjugate vaccine. Clin Infect Dis. 2016;62:1119-25. https://doi.org/10.1093/cid/ciw067

Address for correspondence: Ron Dagan, Pediatric Infectious Disease Unit, Soroka University Medical Center, Beer-Sheva, Israel; e-mail: rdagan@bgu.ac.il

\section{EID podcast Tuberculosis Surveillance and Control in Puerto Rico}

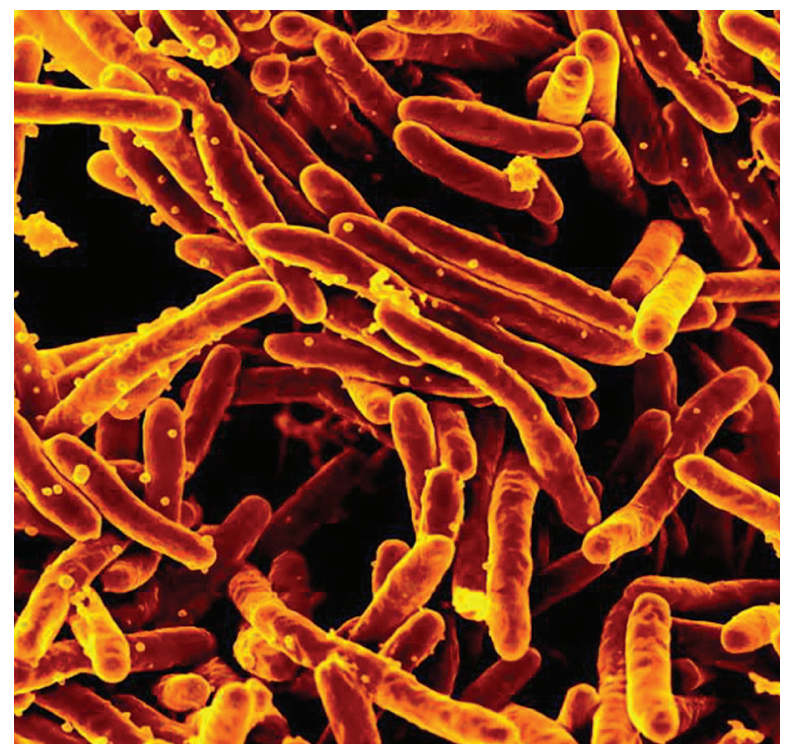

The WHO has recognized Puerto Rico as a promising candidate for the elimination of tuberculosis by 2035 , but many challenges remain before this goal can be achieved. Before going forward, researchers must look back at the historical patterns and developments that have brought them here.

In this EID podcast, Dr. Emilio Dirlikov, a CDC epidemiologist, tells the story of TB surveillance in Puerto Rico from 1898 to 2015.

\section{Visit our website to listen: https: / /go.usa.gov/xysv EMERGING INFECTIOUS DISEASES}

